\title{
Virtual Prototyping at CERN
}

\author{
Silvano de Gennaro
}

CERN, Geneva, Switzerland

\begin{abstract}
This paper describes the activities of the VENUS project at CERN, whose task is to produce Virtual Prototypes of the Large Hadron Collider, and its experimental pits and detectors. These prototypes are made available to the CERN engineers on site, and to the whole HEP community through a 3D graphic WWW browser currently under development.
\end{abstract}

\section{The VENUS Project}

The VENUS [1] (Virtual Environment Navigation in the Underground Sites) project is probably the world's most extensive applications of Virtual Prototypes to Engineering design. Just over one year old, VENUS proposes the latest and most advanced graphic solutions to a number of problems connected with the design of LHC. In order to respond more promptly to the needs of LHC designers and engineers, VENUS features four subprojects:

- Virtual Prototyping

- Territory Impact Study

- Networked Design Integration

- Assembly Planning and Control

We will cover the methods used and results obtained in each of these fields.

\section{Virtual Prototyping}

Virtual Prototyping starts where CAD ends. The CAD objects designed by the CERN engineers are imported into a Virtual Environment, then optimised and treated for their surface properties (colour, material, texture, transparency etc.), and finally organised in a virtual world. The viewer is immediately able to fly through this world and explore it from the inside.

Virtual Prototypes are an ideal replacement for the wooden models traditionally built for the past CERN machines, as they are generated directly from the EUCLID CAD files, therefore they preserve the original accuracy, they can be updated in a matter of minutes, and they allow immersive visualisation, in any preferred scale, through any preferred navigation metaphor. Because the flythrough is performed using one of the numerous off-the shelf packages available on the Virtual Reality market, we are free to add any of the navigation peripherals supported by that package at no extra effort. At present the VENUS lab allows three navigation metaphors:

- Spaceball and computer screen (mono or stereo)

- joystick and video projector (mono or stereo)

- 3-D joystick and VR helmet (mono or stereo, immersive)

The Spaceball is a 6 degrees of freedom, coordinates and velocity input device, allowing easy displacement in any direction in a 3-D environment. This solution is ideal for developing models, as it preserves access to the keyboard, therefore to the package's $\mathrm{X}$-windows interface. On-screen rendering offers higher image quality and can also be selected in stereo mode, using a pair of shutter glasses. 
The second metaphor (joystick and video projector) is used for group visualisation. One person controls the flight through a joystick while a number of viewers can follow on a large stereoscopic screen, wearing polarised glasses. The stereo projector is installed in a conference room adjacent to the VENUS lab, where design work teams meet and discuss the development of the models. The large screen $(160 \times 160 \mathrm{~cm})$ allows more detailed viewing and the stereo vision allows a better understanding of depth and volumes, particularly with semitransparent or wireframed objects.

The third metaphor is the classic Virtual Reality approach to immersive navigation. A VR helmet places you inside the model, giving you a realistic perception of proportions. By moving your head around you can explore the virtual worlds as if you were really walking through it. Translations of your body in the virtual environment are controlled through forward and backward movement buttons on the 3-D joystick.

The LHC Virtual Prototypes are designed in EUCLID, then exported in Wavefront format to VENUS' SGI Onyx RealityEngine2, where they are visualised using Medialab's CLOVIS Virtual Reality package.

\section{Territory Impact Study}

The primary commitment of VENUS at present is to deliver accurate photorealistic 3-D reproductions of the landscape around the access pits that need new surface buildings, such as Point 5 and Point 1, which will host respectively CMS and ATLAS. The purpose of these models is to serve as a basis for a study aimed to minimise the visual impact of the future LHC surface sites on the territory.

The territory elevation curves, as well as the future surface buildings, are modelled in Euclid, then exported the same way as the Virtual Prototypes, to the Onyx. Here the land model is "dressed" with houses, fields, trees, road signs and other details, by pasting textures obtained by scanning photos and post-processing them on a Mac using Adobe Photoshop.

The result is a Virtual World that you can fly through and observe from any possible angle, using the software and metaphors described above. While moving you can also interact with the environment by moving the future buildings horizontally or vertically in real time, and observing the effect on the landscape, until you find the best solution. You can also add at a keyclick hills and forests, or watch the trees grow, in order to establish a planned landscape architecture over time.

\section{Networked Design Integration}

The LHC Virtual Prototypes are physically constrained to the VENUS lab, as they need exclusive hardware and software, available today only in a few other VR laboratories around the world. Therefore they are usable within the scope of the infrastructure design of the pits and the machine, which takes place nearby the VENUS lab, but unfortunately they cannot be made easily available to all the institutes who will design the detectors components.

In order to allow interactive world-wide access to the experiments' 3-D data, VENUS has committed to develop a specialised package to provide web-based, on-screen navigation. The first version of this tool is called i3D [2]. It has been written at CRS4 [3], in Italy, and is available free of charge on the public domain. VENUS will pursue the enhancement of this software in order to use it as a graphic integration tool for LHC.

I3d stands half way between a VR navigation tool and a web browser. As a VR tool it performs on line rendering and navigation, featuring also some of the VR peripherals, such as 
Spaceball and stereo glasses. As a web browser it supports hyperlinks, allowing to download all kinds of objects supported by the web browsers. I3d worlds are loaded via http, in i3D's own format ".3d" or in VRML format ".wrl", ".vrml". VRML [4] stands for "Virtual Reality Markup Language" and is a new standard for VR worlds description. When clicking on a ".i3d", ".wrl" or ".vrml" within a web browser such as Mosaic or Netscape, one triggers the spawn of i3D in a separate window. I3D will then load its world and let you navigate with the look and feel of a VR product. Some of the graphic objects you encounter in the "flight" are bordered in blue. These are hyperlinks to other worlds, or to other web objects. For instance by clicking on a muon detector you may cause Netscape to load the home page of the institute where it is being designed, by clicking on a magnet you can get its technical drawing in a GIF file, and a click on an argon tank can load the whole cryogenics room in your world.

i3D is available at present only on Silicon Graphics stations. Future development of i3D will be carried out at CERN as from September. It includes porting to other UNIX ${ }^{\mathrm{TM}}$ stations and support for engineering features, such as an information window to obtain technical and metric data about the objects.

\section{Assembly Planning and Control}

Although VENUS can be defined mainly as a visualisation lab, its scope goes much beyond the mere visual world. One of the major needs of the engineers in charge of LHC is to plan the operations for the installation of the detectors infrastructures and equipment into the pits.

The ATLAS toroid will be preassembled in items weighing 200 tons each, CMS in three pieces of 1000 tons each. Most of these items must be moved at a maximum acceleration of $0.1 \mathrm{~g}$ and must be aligned with a precision of 6 microns. The operation of lowering these parts into the pits cannot be improvised, but needs careful study and simulation. VENUS is currently evaluating a few software packages that could allow not only to simulate the operations, but also to perform them automatically. After converting our Virtual Prototypes to the standard accepted by these packages, we will be able to let the computer calculate for each item a collision-free path, within the degrees of freedom of our bridge cranes. This path could then be injected into a robot controller that will drive the cranes during the build phases. This should allow us to assemble our future detectors almost without human intervention, therefore minimising the probability of erroneous manoeuvres.

\section{Acknowledgements}

VENUS is a pilot project sponsored by the $\mathrm{CN}$ (Computer and Networks) and $\mathrm{AC}$ (Accelerators Direction) divisions, staffed by myself, plus three part-time members: Nils Høymir, Lars-Johan Rehn and Michele Müller. Many thanks to Damien Riunaud for spending hours scanning mountains, to GianVittorio Frigo for valuable consultancy on Photoshop, and to Chris Jones and David Williams for the enthusiastic support to this project.

\section{References}

[1] http://sgvenus.cern.ch/VENUS/

[2] http://www.crs4.it/ 3diadm/i3d-help/i3d-help.html

[3] http://www.crs4.it/

[4] http://www.sdsc.edu/SDSC/Partners/vrml 